\title{
The zeros of differential-difference polynomials of certain types
}

\author{
Xin Ling Liu' ${ }^{1}$ Li Na Wang ${ }^{2}$ and Kai Liư ${ }^{1 *}$
}

${ }^{*}$ Correspondence: liukai418@126.com

'Department of Mathematics, Nanchang University, Nanchang, Jiangxi 330031, P.R. China Full list of author information is available at the end of the article

\begin{abstract}
In this paper, the zero distribution of differential-difference polynomials $\left[f(z)^{n} f(z+c)\right]^{(k)}$ and $\left[f(z)^{n} \Delta_{c} f\right]^{(k)}$ will be considered. The results can be seen as the differential-difference analogues of Hayman conjecture.
\end{abstract}

MSC: 30D35; 39A05

Keywords: zeros; differential-difference polynomials; Borel exceptional value

\section{Introduction and main results}

In this paper, we use the basic notations of Nevanlinna theory [1,2]. Given a meromorphic function $f(z)$, recall that $\alpha(z) \not \equiv 0, \infty$ is a small function with respect to $f(z)$ if $T(r, \alpha)=$ $S(r, f)$, where $S(r, f)$ is used to denote any quantity satisfying $S(r, f)=o(T(r, f))$, and $r \rightarrow \infty$ outside of a possible exceptional set of finite logarithmic measure.

A Borel exceptional polynomial of $f(z)$ is any polynomial $p(z)$ satisfying

$$
\lambda(f(z)-p(z))=\limsup _{r \rightarrow \infty} \frac{\log ^{+} N\left(r, \frac{1}{f(z)-p(z)}\right)}{\log r}<\rho(f),
$$

where $\lambda(f(z)-p(z))$ is the exponent of the convergence of zeros of $f(z)-p(z)$ and $\rho(f)$ is the order of $f(z)$. In the following, we assume that $c$ is a nonzero complex constant, $n$ and $k$ are positive integers unless otherwise specified.

The zero distribution of differential polynomials is a classical topic in the theory of meromorphic functions. Hayman [3, Theorem 10] firstly considered the value distribution of $f^{n} f^{\prime}-1$, where $f$ is a transcendental function. Then later this topic was considered by several authors such as $[4,5]$.

Theorem A ([5, Theorem 1]) Let $f$ be a transcendental meromorphic function. If $n \geq 1$, then $f^{n} f^{\prime}-1$ has infinitely many zeros.

Since $f^{n} f^{\prime}$ can be written as $\frac{\left(f^{n+1}\right)^{\prime}}{n+1}$, Wang and Fang [6] improved Theorem A by proving the following result.

Theorem B ([6, Corollary 1]) Letf be a transcendental meromorphic function. If $n \geq k+1$, then $\left(f^{n}\right)^{(k)}-1$ has infinitely many zeros.

The difference logarithmic derivative lemma, given by Chiang and Feng [7, Corollary 2.5], Halburd and Korhonen [8, Theorem 2.1], [9, Theorem 5.6], plays an important

\section{Springer}

(c) 2012 Liu et al.; licensee Springer. This is an Open Access article distributed under the terms of the Creative Commons Attribution License (http://creativecommons.org/licenses/by/2.0), which permits unrestricted use, distribution, and reproduction in any medium, provided the original work is properly cited. 
part in considering the difference analogues of Nevanlinna theory. With the development of difference analogue of Nevanlinna theory, many authors paid their attention to the zero distribution of difference polynomials [10-18]. Laine and Yang [12, Theorem 2] firstly considered the zero distribution of $f(z)^{n} f(z+c)-a$, where $a$ is a nonzero constant, Liu and Yang [13, Theorems 1.2 and 1.4] also considered the zeros of $f(z)^{n} f(z+c)-p(z)$ and $f(z)^{n} \Delta_{c} f-p(z)$, where $\Delta_{c} f:=f(z+c)-f(z)$ and $p(z)$ is a nonzero polynomial. These results are summarized in Theorem $C$ below, and they can be seen as difference analogues of Theorem A.

Theorem C Let $f$ be a transcendental entire function of finite order and $p(z)$ be a nonzero polynomial. If $n \geq 2$, then $f(z)^{n} f(z+c)-p(z)$ has infinitely many zeros. If $f$ is not a periodic function with period $c$ and $n \geq 2$, then $f(z)^{n} \Delta_{c} f-p(z)$ has infinitely many zeros.

As the results on the difference analogues of Theorem B, Liu, Liu and Cao [14] investigated the zeros of $\left[f(z)^{n} f(z+c)\right]^{(k)}-\alpha(z)$ and $\left[f(z)^{n} \Delta_{c} f\right]^{(k)}-\alpha(z)$, where $\alpha(z)$ is a nonzero small function with respect to $f(z)$. Some results can also be found in [16] on the case that $\alpha(z)$ is a nonzero polynomial.

Theorem D ([14, Theorems 1.1 and 1.3]) Let $f$ be a transcendental entire function of finite order and $\alpha(z)$ be a nonzero small function with respect to $f(z)$. If $n \geq k+2$, then $\left[f(z)^{n} f(z+\right.$ $c)]^{(k)}-\alpha(z)$ has infinitely many zeros. If $f$ is not a periodic function with period $c$ and $n \geq$ $k+3$, then $\left[f(z)^{n} \Delta_{c} f\right]^{(k)}-\alpha(z)$ has infinitely many zeros.

However, we remark that the zeros of $\left[f(z)^{n} f(z+c)\right]^{(k)}$ or $\left[f(z)^{n} \Delta_{c} f\right]^{(k)}$ were not mentioned in $[14,16]$. We will consider this problem in this paper, some ideas of proofs partially relying on the ideas used in $[14,16]$.

It is easy to know that if $f$ has infinitely many zeros, then $f(z)^{n} f(z+c)$ must have infinitely many zeros. In fact, we mainly get some results on the case that $n=1$. The following example shows that $f(z) f(z+c)$ can admit infinitely many zeros, however, $[f(z) f(z+c)]^{(k)}$ has finitely many zeros.

Example 1 Suppose that $e^{c}=-1$ and $f(z)=e^{z}+1$. Then $f(z) f(z+c)=1-e^{2 z}$ has infinitely many zeros, but $[f(z) f(z+c)]^{(k)}=-2^{k} e^{2 z}$ has no zeros.

What conditions will guarantee that $[f(z) f(z+c)]^{(k)}$ can admit infinitely many zeros? Obviously, the value 1 is the Borel exceptional value of $f(z)$ in Example 1. Here, we obtain the following result.

Theorem 1.1 Let $f$ be a finite order transcendental entire function with a Borel exceptional polynomial $q(z)$. Then the following statements hold.

(i) If $q(z) \equiv 0$, then $[f(z) f(z+c)]^{(k)}$ has no nonzero Borel exceptional value.

(ii) If $q(z) \not \equiv 0$ and $\operatorname{deg}(q(z))<\frac{k}{2}$, then $[f(z) f(z+c)]^{(k)}$ has infinitely many zeros, except in the case $f(z)=A q(z) e^{\alpha z}+q(z)$ and $e^{c}=-1$, where $A$ is a nonzero constant.

(iii) If $\operatorname{deg}(q(z)) \geq \frac{k}{2}$, then $[f(z) f(z+c)]^{(k)}$ has infinitely many zeros.

Remark 1 (1) If $q(z) \equiv 0$, then $[f(z) f(z+c)]^{(k)}$ can admit finitely or infinitely many zeros. For example, if $f(z)=z e^{z}$ and $e^{c}=2$, then $[f(z) f(z+c)]^{(k)}=p(z) e^{2 z}$ has finitely many zeros, 
where $p(z)$ is a polynomial in $z$. If $f(z)=\left(e^{z}+1\right) e^{z^{2}}$ and $e^{c}=-1$, thus the value 0 is a Borel exceptional value of $f(z)$, then $[f(z) f(z+c)]^{\prime}=\left[(4 z+2 c)-(5 z+2 c) e^{2 z}\right] e^{z^{2}+(z+c)^{2}}$ has infinitely many zeros, but $\lambda\left([f(z) f(z+c)]^{\prime}\right)=1<\rho\left([f(z) f(z+c)]^{\prime}\right)=2$.

(2) From Example 1, we know that the exceptional case in (ii) can occur.

If $f$ has finitely many zeros, then $f(z)^{n} f(z+c)$ must have finitely many zeros; however, $f(z)^{n} \Delta_{c} f$ can admit infinitely many zeros. The following example shows that the zero distribution of $f(z)^{n} \Delta_{c} f$ is different from that of $f(z)^{n} f(z+c)$.

Example 2 Suppose that $e^{c}=-1$ and $f(z)=e^{z^{2}}$. Then $f(z)^{n} f(z+c)$ has no zeros, but $f(z)^{n} \Delta_{c} f=e^{(n+1) z^{2}}\left(e^{2 z c+c^{2}}-1\right)$ has infinitely many zeros.

Chen [10] investigated the problem: what conditions will guarantee that $f(z)^{n} \Delta_{c} f$ have infinitely many zeros. From the following Example 3, we know that the zero distribution of $\left[f(z)^{n} \Delta_{f} f\right]^{(k)}$ may be different from that of $f(z)^{n} \Delta_{f} f$.

Example 3 Suppose that $f(z)=e^{z}+z$ of $\rho(f)=1$ and $e^{c}=1$. Then $f(z) \Delta_{c} f=c\left(e^{z}+z\right)$ has infinitely many zeros, but $\left[f(z) \Delta_{f} f\right]^{(k)}=c e^{z}$ has no zeros, where $k \geq 2$.

Thus, it is natural to consider what conditions can guarantee that $\left[f(z)^{n} \Delta_{c} f\right]^{(k)}$ have infinitely many zeros. We obtain the following theorems.

Theorem 1.2 Let $f$ be a transcendental entire function with finite order, $n \geq 2, \Delta_{c} f \not \equiv 0$. If $f(z)$ has finitely many zeros and $\rho(f) \neq 1$, then $\left[f(z)^{n} \Delta_{c} f\right]^{(k)}$ has infinitely many zeros. If $f(z)$ has finitely many zeros and $\rho(f)=1$, then $\left[f(z)^{n} \Delta_{c} f\right]^{(k)}$ has finitely many zeros.

In the case of $n=1$, by using a similar method as in the proof of Theorem 1.1, we have the following result.

Theorem 1.3 Let $f$ be a finite-order transcendental entire function with a Borel exceptional polynomial $q(z), k$ be a positive integer, $\Delta_{c} f \not \equiv 0$. Then the following statements hold.

(i) If $q(z) \equiv 0$, then $\left[f(z) \Delta_{c} f\right]^{(k)}$ has no nonzero Borel exceptional value.

(ii) If $q(z) \not \equiv 0$ and $\operatorname{deg} q(z)<\frac{k+1}{2}$, then $\left[f(z) \Delta_{f} f\right]^{(k)}$ has infinitely many zeros, except in the case $f(z)=A e^{\alpha z}+q(z)$ and $e^{c}=1$, where $q(z)$ is not a constant and $A$ is a nonzero constant.

(iii) If $\operatorname{deg} q(z) \geq \frac{k+1}{2}$, then $\left[f(z) \Delta_{f} f\right]^{(k)}$ has infinitely many zeros.

Remark 2 From Example 3, we know that the exceptional case in (ii) can occur.

\section{Some lemmas}

The first lemma is the characteristic function relationship between $f(z)$ and $f(z+c)$, provided that $f(z)$ is a transcendental meromorphic function of finite order.

Lemma 2.1 ([7, Theorem 2.1]) Let $f(z)$ be a transcendental meromorphic function of finite order. Then

$$
T(r, f(z+c))=T(r, f)+S(r, f) .
$$


From (2.1) and the classical result of Nevanlinna theory, we know that $\rho(f)=\rho(f(z+c))=$ $\rho\left(f^{(k)}\right)$.

Following Hayman [19, pp.75-76], we define an $\varepsilon$-set $E$ to be a countable union of discs not containing the origin and subtending angles at the origin whose sum is finite. If $E$ is an $\varepsilon$-set, then the set of $r \geq 1$ for which the circle $S(0, r)$ meets $E$ has finite logarithmic measure.

Lemma 2.2 ([20]) Let $g(z)$ be a transcendental meromorphic function of $\rho(g)<1, h>0$. Then there exists an $\varepsilon$-set $E$ such that

$$
\frac{g^{\prime}(z+c)}{g(z+c)} \rightarrow 0 \quad \text { and } \quad \frac{g(z+c)}{g(z)} \rightarrow 1 \quad \text { as } z \rightarrow \infty \text { in } \mathbb{C} \backslash E,
$$

uniformly in $c$ for $|c| \leq h$. Further, $E$ may be chosen so that for large $z \notin E$, the function $g$ has no zeros or poles in $|\zeta-z| \leq h$.

Lemma 2.3 ([2, Theorem 1.62]) Let $f_{j}(z)$ be meromorphic functions, $f_{k}(z)(k=1,2, \ldots, n-1)$ be not constants, satisfying $\sum_{j=1}^{n} f_{j}=1$ and $n \geq 3$. If $f_{n}(z) \not \equiv 0$, and

$$
\sum_{j=1}^{n} N\left(r, \frac{1}{f_{j}}\right)+(n-1) \sum_{j=1}^{n} \bar{N}\left(r, f_{j}\right)<(\lambda+o(1)) T\left(r, f_{k}\right)
$$

where $\lambda<1, k=1,2, \ldots, n-1$, then $f_{n}(z) \equiv 1$.

Lemma 2.4 ([2, Theorem 1.51]) Let $f_{j}(z)(j=1,2, \ldots, n)(n \geq 2)$ be meromorphic functions, $g_{j}(z)(j=1,2, \ldots, n)$ be entire functions satisfying

(i) $\sum_{j=1}^{n} f_{j}(z) e^{g_{j}(z)} \equiv 0$,

(ii) when $1 \leq j<k \leq n, g_{j}(z)-g_{k}(z)$ is not a constant,

(iii) when $1 \leq j \leq n, 1 \leq h<k \leq n, T\left(r, f_{j}\right)=o\left(T\left(r, e^{g_{h}-g_{k}}\right)\right)(r \rightarrow \infty, r \notin E)$, where $E \subset(1, \infty)$ is of finite linear measure or finite logarithmic measure. Then $f_{j}(z) \equiv 0$ $(j=1,2, \ldots, n)$.

For the proofs of Theorems 1.1 and 1.3, we need the following results, which are related to the growth of solutions of linear difference equations. Here, we give the versions with small changes of the type of equations; the proofs are similar.

Lemma 2.5 ([7, Theorem 9.2]) Let $A_{0}(z), \ldots, A_{n}(z)$ be entire functions such that there exists an integer $l, 0 \leq l \leq n$, such that

$$
\rho\left(A_{l}(z)\right)>\max _{0 \leq j \leq n, j \neq l} \rho\left(A_{j}(z)\right) .
$$

Iff $(z)$ is a nontrivial meromorphic solution of the equation

$$
A_{n}(z) y\left(z+c_{n}\right)+\cdots+A_{1}(z) y\left(z+c_{1}\right)+A_{0}(z) y(z)=0,
$$

then $\rho(f) \geq \rho\left(A_{l}(z)\right)+1$. 
Lemma $2.6\left(\left[21\right.\right.$, Theorem 1.2]) Let $P_{0}(z), \ldots, P_{n}(z)$ be polynomials, $P_{n}(z) P_{0}(z) \not \equiv 0$ and satisfy

$$
\operatorname{deg}\left(P_{n}(z)+\cdots+P_{0}(z)\right)=\max \left\{\operatorname{deg} P_{j}(z): j=0, \ldots, n\right\} \geq 1 .
$$

Then every finite order meromorphic solution $f(z)(\not \equiv 0)$ of the equation

$$
P_{n}(z) f\left(z+c_{n}\right)+\cdots+P_{1}(z) f\left(z+c_{1}\right)+P_{0}(z) f(z)=0
$$

satisfies $\rho(f) \geq 1$.

\section{Proofs of Theorem 1.1 and Theorem 1.3}

The ideas for the proof of Theorem 1.1 and Theorem 1.3 are similar, here we just give a complete proof of Theorem 1.1.

Since $q(z)$ is a Borel exceptional polynomial of $f(z)$, the transcendental entire function $f(z)$ with finite order can be written as $f(z)=q(z)+h(z) e^{\alpha z^{s}}$, where $\alpha$ is a nonzero constant and $h(z)$ is a nonzero entire function with $\lambda(h) \leq \rho(h)<\rho(f)=s$. Hence,

$$
f(z+c)=q(z+c)+h(z+c) e^{\alpha(z+c)^{s}}=q(z+c)+h_{1}(z) e^{\alpha z^{s}},
$$

where

$$
h_{1}(z)=h(z+c) e^{\alpha\left(C_{s}^{1} z^{s-1} c+C_{s}^{2} z^{s-2} c^{2}+\cdots+C_{s}^{s-1} z c^{s-1}+c^{s}\right)},
$$

and $\rho\left(h_{1}\right)<\rho(f)=s$. Assume that $[f(z) f(z+c)]^{(k)}$ has finitely many zeros. Then from Hadamard's factorization theorem and Lemma 2.1, we have

$$
[f(z) f(z+c)]^{(k)}=A(z) e^{\beta z^{s}}
$$

where $A(z)$ is an entire function with finitely many zeros and $\rho(A)<s, \beta$ is a nonzero constant. For any positive integer $k$, from (3.1) and (3.3), we get

$$
\left[\left(q(z)+h(z) e^{\alpha z^{s}}\right)\left(q(z+c)+h_{1}(z) e^{\alpha z^{s}}\right)\right]^{(k)}-A(z) e^{\beta z^{s}}=0,
$$

which implies that

$$
\begin{aligned}
& {\left[q(z) q(z+c)+q(z) h_{1}(z) e^{\alpha z^{s}}+q(z+c) h(z) e^{\alpha z^{s}}\right.} \\
& \left.\quad+h(z) h_{1}(z) e^{2 \alpha z^{s}}\right]^{(k)}-A(z) e^{\beta z^{s}}=0
\end{aligned}
$$

Thus, we get

$$
D_{1}(z) e^{\alpha z^{s}}+D_{2}(z) e^{2 \alpha z^{s}}-A(z) e^{\beta z^{s}}=-[q(z) q(z+c)]^{(k)},
$$

where $D_{j}(z)$ are differential polynomials of $h(z), h_{1}(z), q(z), q(z+c)$ and $\rho\left(D_{j}(z)\right)<s, j=1,2$.

Case (i). If $q(z) \equiv 0$, then $[f(z) f(z+c)]^{(k)}=D_{2}(z) e^{2 \alpha z^{s}}$, where $\rho\left(D_{2}(z)\right)<s$. This implies that the value 0 is a Borel exceptional value of $[f(z) f(z+c)]^{(k)}$. Since entire functions have 
at most one Borel exceptional value, the function $[f(z) f(z+c)]^{(k)}$ has no nonzero finite Borel exceptional value.

Case (ii). If $\operatorname{deg}(q(z))<\frac{k}{2}$ and $q(z) \not \equiv 0$, then $[q(z) q(z+c)]^{(k)} \equiv 0$. From Lemma 2.4, we get $D_{1}(z) \equiv 0$ and $D_{2}(z)-A(z) \equiv 0$. We will prove that $B_{1}(z):=q(z) h_{1}(z)+q(z+c) h(z) \equiv 0$, where $\rho\left(B_{1}(z)\right)<s$. If $k=1$, then

$$
B_{1}^{\prime}(z)+\alpha s z^{s-1} B_{1}(z)=0
$$

Thus, the nontrivial solution $B_{1}(z)$ of differential equation (3.7) satisfies $\rho\left(B_{1}(z)\right)=s$, which is a contradiction with $\rho\left(B_{1}(z)\right)<s$, thus $B_{1}(z) \equiv 0$. If $k=2$, let $g(z)=B_{1}^{\prime}(z)+\alpha s z^{s-1} B_{1}(z)$. Then

$$
g^{\prime}(z)+\alpha s z^{s-1} g(z)=0
$$

This implies that $\rho(g(z))=s$, a contradiction with $\rho(g(z)) \leq \rho\left(B_{1}(z)\right)<s$. Thus $g(z) \equiv 0$ and $B_{1}(z) \equiv 0$. Using this method for any positive integer $k$, we can get $B_{1}(z) \equiv 0$, and so

$$
q(z+c) h(z)+q(z) h(z+c) e^{\alpha\left(C_{s}^{1} z^{s-1} c+C_{s}^{2} z^{s-2} c^{2}+\cdots+C_{s}^{s-1} z c^{s-1}+c^{s}\right)} \equiv 0 .
$$

We get $s=1$, from Lemma 2.5 and $\rho(h)<s$, thus the above equation implies that

$$
q(z+c) h(z)+q(z) h(z+c) e^{\alpha c} \equiv 0 .
$$

Combining (3.9), $\rho(h)<s=1$ with Lemma 2.6, we get the degree of $q(z+c)+q(z) e^{\alpha c}$ must be less than the degree of $q(z)$ if $q(z)$ is a nonconstant polynomial or $q(z)$ is a constant. If $q(z)$ is a nonconstant polynomial, then $e^{\alpha c}=-1$. Hence,

$$
q(z+c) h(z)-q(z) h(z+c) \equiv 0,
$$

which implies that $\frac{h(z+c)}{q(z+c)}=\frac{h(z)}{q(z)}$, thus $H(z)=\frac{h(z)}{q(z)}$ is a periodic function with period $c$. Since $\rho(H)=\rho(h)<1$, thus $H(z)$ must be a constant $A$, which implies that $h(z)$ must be a polynomial of the form $h(z)=A q(z)$.

If $q(z)$ is a constant, we have $h(z)+h(z+c) e^{\alpha c} \equiv 0$. From Lemma 2.2 and $\rho(h)<s=1$, we get $e^{\alpha c}=-1$. Thus $h(z)$ must be a constant, hence $h(z)$ and $q(z)$ are both constants, and so we may write $h(z)=A q(z)$.

Case (iii). If $\operatorname{deg}(q(z)) \geq \frac{k}{2}$, then $[q(z) q(z+c)]^{(k)} \not \equiv 0$. Combining $\rho\left(D_{j}(z)\right)<s, j=1,2$, $\rho(A(z))<s$ with (3.6) and Lemma 2.3, we get

$$
\left[D_{2}(z)-A(z)\right] e^{2 \alpha z^{s}}=-[q(z) q(z+c)]^{(k)}
$$

or

$$
D_{1}(z) e^{\alpha z^{s}}=-[q(z) q(z+c)]^{(k)}
$$

which is impossible. Thus $\left[f(z)^{n} f(z+c)\right]^{(k)}$ has infinitely many zeros. The proof of Theorem 1.1 is completed. 


\section{Proof of Theorem 1.2}

Suppose that $\rho(f) \neq 1$ and $f$ has finitely many zeros. Thus, from Hadamard's factorization theorem, $f(z)$ can be written as $f(z)=g(z) e^{h(z)}$, where $g(z) \not \equiv 0, h(z)$ are polynomials, and $\operatorname{deg}(h(z)) \geq 2$. Suppose that $\left[f(z)^{n} \Delta_{c} f\right]^{(k)}$ has finitely many zeros. Then

$$
\left[g(z)^{n} g(z+c) e^{n h(z)+h(z+c)}-g(z)^{n+1} e^{(n+1) h(z)}\right]^{(k)}=g_{1}(z) e^{h_{1}(z)},
$$

which implies that

$$
g_{2}(z) e^{n h(z)+h(z+c)}+g_{3}(z) e^{(n+1) h(z)}-g_{1}(z) e^{h_{1}(z)}=0,
$$

where $g_{2}(z), g_{3}(z)$ are nonzero polynomials.

To avoid a contradiction with Lemma 2.4, we get $n h(z)+h(z+c)-h_{1}(z)$ and $(n+1) h(z)-$ $h_{1}(z)$ are constants. Thus, we get $h(z+c)-h(z)$ is a constant, then $\operatorname{deg}(h(z))=1$, a contradiction with $\operatorname{deg}(h(z)) \geq 2$.

If $f$ has finitely many zeros and $\rho(f)=1$, which implies that $f(z)=p(z) e^{b z}$. Thus, $\left[f(z)^{n} \Delta_{f} f\right]^{(k)}=p_{1}(z) e^{(n+1) b z}$ has finitely many zeros, where $p_{1}(z)$ is a nonzero polynomial. Thus, we have completed the proof of Theorem 1.2.

\section{Competing interests}

The authors declare that they have no competing interests.

\section{Authors' contributions}

The authors have achieved equal contributions to this paper. All authors read and approved the final version of the manuscript.

\section{Author details}

${ }^{1}$ Department of Mathematics, Nanchang University, Nanchang, Jiangxi 330031, P.R. China. ${ }^{2}$ Department of Sciences, Nanchang Institute of Technology, Nanchang, Jiangxi 330039, P.R. China.

\section{Acknowledgements}

The authors would like to thank the referees for valuable suggestions for improving our paper. This work was partially supported by the NSFC (No. 11101201), the NSF of Jiangxi (No. 2010GQS0144).

Received: 27 May 2012 Accepted: 4 September 2012 Published: 19 September 2012

\section{References}

1. Laine, I: Nevanlinna Theory and Complex Differential Equation. Studies in Mathematics, vol. 15. de Gruyter, Berlin (1993)

2. Yang, CC, Yi, HX: Uniqueness Theory of Meromorphic Functions. Kluwer Academic, Dordrecht (2003)

3. Hayman, WK: Picard values of meromorphic functions and their derivatives. Ann. Math. 70, 9-42 (1959)

4. Bergweiler, W, Eremenko, A: On the singularities of the inverse to a meromorphic function of finite order. Rev. Mat. Iberoam. 11, 355-373 (1995)

5. Chen, HH, Fang, ML: On the value distribution of $f^{n} f$. Sci. China Ser. A 38, 789-798 (1995)

6. Wang, YF, Fang, ML: Picard values and normal families of meromorphic functions with multiple zeros. Acta Math. Sin. 14(1), 17-26 (1998)

7. Chiang, YM, Feng, SJ: On the Nevanlinna characteristic $f(z+\eta)$ and difference equations in the complex plane. Ramanujan J. 16, 105-129 (2008)

8. Halburd, RG, Korhonen, RJ: Difference analogue of the lemma on the logarithmic derivative with applications to difference equations. J. Math. Anal. Appl. 314, 477-487 (2006)

9. Halburd, RG, Korhonen, RJ: Meromorphic solutions of difference equations, integrability and the discrete Painlevé equations. J. Phys. A 40, 1-38 (2007)

10. Chen, ZX: Value distribution of products of meromorphic functions and their differences. Taiwan. J. Math. 15, 1411-1421 (2011)

11. Huang, ZB, Chen, ZX: A Clunie lemma for difference and q-difference polynomials. Bull. Aust. Math. Soc. 81, 23-32 (2010)

12. Laine, I, Yang, CC: Value distribution of difference polynomials. Proc. Jpn. Acad., Ser. A, Math. Sci. 83, 148-151 (2007)

13. Liu, K, Yang, LZ: Value distribution of the difference operator. Arch. Math. 92, 270-278 (2009)

14. Liu, K, Liu, XL, Cao, TB: Some results on zeros and uniqueness of difference-differential polynomials. Appl. Math. J. Chin. Univ. Ser. A 27, 94-104 (2012) 
15. Liu, K, Liu, XL, Cao, TB: Value distributions and uniqueness of difference polynomials. Adv. Differ. Equ. 2011, Article ID $234215(2011)$

16. Liu, K, Cao, TB, Liu, XL: The properties on differential-difference polynomials (submitted)

17. Qi, XG, Dou, J, Yang, LZ: Uniqueness and value distribution for difference operators of meromorphic function. Adv. Differ. Equ. 2012, 32 (2012)

18. Zhang, JL: Value distribution and shared sets of differences of meromorphic functions. J. Math. Anal. Appl. 367 , 401-408 (2010)

19. Hayman, WK: Slowly growing integral and subharmonic functions. Comment. Math. Helv. 34, $75-84$ (1960)

20. Bergweiler, W, Langley, JK: Zeros of difference of meromorphic functions. Math. Proc. Camb. Philos. Soc. 142, 133-147 (2007)

21. Chen, ZX: Growth and zeros of meromorphic solution of some linear difference equations. J. Math. Anal. Appl. 373, 235-241 (2011)

doi:10.1186/1687-1847-2012-164

Cite this article as: Liu et al.: The zeros of differential-difference polynomials of certain types. Advances in Difference Equations 2012 2012:164.

Submit your manuscript to a SpringerOpen ${ }^{\circ}$ journal and benefit from:

- Convenient online submission

- Rigorous peer review

- Immediate publication on acceptance

- Open access: articles freely available online

- High visibility within the field

- Retaining the copyright to your article

Submit your next manuscript at $>$ springeropen.com 